\title{
Civilisations
}

Revue internationale d'anthropologie et de sciences

humaines

$51 \mid 2004$

Religions transnationales

\section{A propos des notions de globalisation et de transnationalisation}

Stefania Capone

\section{OpenEdition \\ Journals}

Édition électronique

URL : http://journals.openedition.org/civilisations/634

DOI : $10.4000 /$ civilisations. 634

ISSN : 2032-0442

\section{Éditeur}

Institut de sociologie de l'Université Libre de Bruxelles

\section{Édition imprimée}

Date de publication : 1 janvier 2004

Pagination : 9-22

ISSN : 0009-8140

\section{Référence électronique}

Stefania Capone, "A propos des notions de globalisation et de transnationalisation », Civilisations [En ligne], 51 | 2004, mis en ligne le 06 janvier 2009, consulté le 01 mai 2019. URL : http:// journals.openedition.org/civilisations/634; DOI : 10.4000/civilisations.634 


\section{A propos des notions de globalisation et de transnationalisation}

\section{STEFANIA CAPONE}

Depuis quelques années, dans les revues d'anthropologie françaises, quelques rares auteurs se font l'écho des débats, particulièrement animés outre-atlantique, sur les phénomènes de globalisation culturelle (voir, entre autres, Assayag et Bénëi, 2000 et Friedman, 2000). Certains, comme Jean-Loup Amselle, voient dans le thème de la globalisation culturelle « un véritable cul-de-sac pour l'anthropologie » (2001: 18). En fait, si, comme les anthropologues postmodernes l'affirment, notre époque est radicalement différente de toutes celles qui l'ont précédée, au sens où la contemporanéité consisterait en une mise en relation généralisée et une interdépendance totale des cultures de la planète, cela impliquerait l'existence d'une phase de l'histoire de l'humanité au cours de laquelle certaines sociétés vivaient repliées sur elles-mêmes, formant de véritables sociétés closes. Or, selon cet auteur, plusieurs exemples, et notamment celui concernant le système du kula, sorte de phénomène de globalisation « primaire ou partiel » (ibid. : 37), montrent comment le concept d'auto-subsistance, appliqué aux sociétés primitives, peut être trompeur. Il n'existerait donc pas de véritable rupture entre l'objet passé de l'anthropologie et son objet actuel. En réalité, plus que la globalisation qui aurait radicalement transformé les sociétés primitives et aurait dépossédé les anthropologues de leur objet, c'est le regard porté par l'enquêteur sur son terrain qui serait, aujourd'hui, fondamentalement nouveau.

Cependant, ces critiques ne sont pas totalement inédites. James Clifford avait déjà souligné, en 1997, la continuité entre différentes phases de globalisation culturelle, qui ne présupposent, en aucun cas, la préexistence de sociétés closes : "The new paradigms begin with historical contact, with entanglement at intersecting regional, national, and transnational levels. Contact approaches presuppose not sociocultural wholes subsequently brought into relationship, but rather systems already constituted relationally, entering new relations through historical processes of displacement " (Clifford, 1997 : 7). De même, Ulf Hannerz écrivait en 1996 : "That image of cultural mosaic, where each culture would have been a territorial entity with clear, sharp, 
enduring edges, never really corresponded with realities. There were always interactions, and a diffusion of ideas, habits, and things » (1996:18).

Bien évidemment, la notion de globalisation n'est pas nouvelle, car elle est, pour ne donner qu'un exemple, intrinsèque aux religions à vocation universelle, comme l'islam ou le christianisme. Qu'y aurait-il alors de véritablement nouveau dans cette idée d'une « culture globale »? Pour Anthony D. Smith, c'est le phénomène de la déterritorialisation qui rend cette nouvelle culture profondément différente de celles qui l'ont précédée. L'hellénisation ou la pax romana étaient des « impérialismes culturels pré-modernes » qui demeuraient liés à leurs lieux d'origine. Or, la culture globale contemporaine ne serait, elle, liée à aucun lieu déterminé (Smith, 1998 : 189).

\section{DE LA GLOBALISATION À LA TRANSNATIONALISATION}

Selon certains auteurs, l'anthropologie contemporaine américaine concevrait la théorie de la globalisation comme le résultat d'une réorganisation sociale et spatiotemporelle. Pour Jackie Assayag (1998a : 204), « c'est à elle qu'est dévolue la tâche d'éclairer la reconfiguration des identités individuelles ou des communautés au sein d'un monde devenu transnational ». Mais existe-t-il une relation de cause à effet entre le phénomène de globalisation et l'avènement d'un monde transnational? Selon Martin Albrow (1996), il n'y aurait pas de connexion intrinsèque entre ces deux phénomènes, dont la rencontre ne constitue qu'un « événement historique contingent ». Peter van der Veer (2001) semble partager le même point de vue, lorsqu'il analyse les rapports entre les notions de "nation » et de "trans-nation »: "Processes of globalization have been intrinsic to processes of state-formation both in colonizing and colonized societies. This is not taken sufficiently into account in theories of globalization that posit the dissolution of the nation-state today as a consequence of the development of transnational governance and the global economy ".

Cette disjonction entre deux notions trop souvent associées est également présente dans les écrits de Ulf Hannerz : "I am also somewhat uncomfortable with the rather prodigious use of the term globalization to describe just about any process or relationship that somehow crosses state boundaries. [...] The term 'transnational' is in a way more humble, and often a more adequate label for phenomena which can be of quite variable scale and distribution, even when they do share the characteristic of not being contained within a state » (Hannerz, $1996: 6$ ).

Mais, qu'est-ce qui fait aujourd'hui la nouveauté de cette rencontre? Ce que Peter van der Veer (2001) appelle la «mort de la distance». Au lieu de communautés de migrants qui essaient désespérément de garder le contact avec leur lieu d'origine, il y aurait constitution de réseaux diasporiques. Selon d'autres auteurs, les nouvelles technologies (électroniques et multimédias) contribuent à présent à l'intensification de « réseaux culturels déterritorialisés » (Clarke, 2004, sous presse). S'agirait-il d'une véritable révolution? Ce n'est pas ce que semble penser Bruno Latour (1991) qui voit là une simple conséquence de l'expansion historique de l'économie capitaliste.

Jonathan Friedman fait une critique acérée des discours transnationaux qui relèveraient davantage d'un projet idéologique que d'une découverte scientifique, «programme élitiste dont la vision d'en haut, s'appuie [...] sur l'expérience du vol aérien » (2000 : 202-203). L'anthropologie transnationale serait ainsi, pour lui, le fait d'« intel- 
lectuels en diaspora $\rangle^{1}$. Au contraire, on ne peut que partager le point de vue de Peter Beyer (1998 : 18), lorsqu'il souligne que « la communication globale » des migrants est considérable et n'est, en aucun cas, limitée à « certaines élites privilégiées ». Nous ajouterons que les communautés de migrants ne sont pas les seuls concernées, et que l'on peut tisser des réseaux transnationaux tout en restant chez soi.

\section{ENTRE DÉTERRITORIALISATION ET RETERRITORIALISATION}

La notion de déterritorialisation est particulièrement prise à parti par certains auteurs. C'est le cas de Jonathan Friedman, pour qui cette notion relèverait de ce qu'il appelle « la vulgate transnationale » (2000 : 193). Cette version simplifiée de théories bien plus complexes, comme celle de James Clifford, ferait coïncider la métaphore de la racine avec la catégorie d'Etat-nation, « représenté comme une unité close, à la population homogène et au mode de fonctionnement dominé précisément par la délimitation même, par la territorialité et, donc par l'exclusion » (ibid.). Cette réduction de l'Etat-nation à un ensemble culturel isolé mettrait ainsi en scène les «notions de pureté nationale, d'absolutisme ethnique et toutes les formes d'essentialisme » (ibid. $)^{2}$. On peut se demander si les «tenants du transnationalisme » envisagent vraiment de cette façon leur travail, lorsqu'ils analysent l'effacement de tout ancrage d'une culture dans un territoire. Friedman (ibid. : 190) y voit la conséquence de ce qu'il définit comme « l'ancrage biographique des objets intellectuels », à savoir « l'assimilation des objets culturels aux existences humaines », une assimilation qui conduirait, selon lui, à de graves confusions : «C'est, paradoxalement, le caractère limité de l'approche transnationale, son aversion pour le local et la fermeture, qui conduit ses tenants à critiquer ceux qui parlent de limites et de territorialisation, termes considérés comme dépassés et même réactionnaires » (ibid. : 199).

Or, le processus de déterritorialisation se fait rarement sans qu'il y ait reterritorialisation à la suite. S'il y a dissolution ou déplacement des points de référence, des racines ou des frontières, il y a aussi production parallèle de discours sur les origines qui permettent de « réancrer » ce qui a été « déterritorialisé » dans de nouveaux espaces, réels ou symboliques. Ainsi, Kamari Clarke, analysant le cas d'Oyotunji Village aux Etats-Unis, parle du « renforcement des attaches nationales ou régionales à des lieux ou des discours sur les origines », un processus parallèle à l'intensification d'alliances transnationales : "[...] even in the midst of globalization, what persists is the strengthening, rather than the weakening, of attitudes toward territorialized origines -autochthonous attachments. In other words, 'roots'narratives, as they are used by people to align identity with localities of birth, reinforce particular modernities of blood and nationhood. The dilemma, therefore, especially given the disjunctures in fluidity and fixity, is in understanding how originary narratives, which exist alongside transnational movements, circulate and continue to be maintained within new lines of

1. Assayag et Bénéï (2000 : 18), dans l'introduction du numéro spécial de L'Homme consacré aux « intellectuels en diaspora », écrivaient qu' « il est frappant de constater qu'une grande part des travaux, consacrés à ce domaine et livrés à la connaissance de la communauté scientifique internationale, sont l'œuvre d'auteurs d'origine sud-asiatique » résidant aux Etats-Unis.

2. Voir aussi la critique en ce sens de Jean-Loup Amselle (2000). 
global authority, be they religious or legal, political or cultural » (Clarke, 2004, sous presse). Le rapport, souvent conflictuel, entre « racine » et " rhizome » met en scène une nouvelle façon de penser le local, qui produit des discours concurrents sur les origines. En réalité, le passage du global au local met en lumière une zone de tension où des codes multiples de savoir entrent en contact, engendrant des processus à la fois d'homogénéisation et de différenciation. Dans le champ religieux, cette tension s'exprime souvent par le(s) discours sur la tradition, permettant à chaque groupe, en quête de légitimation et de suprématie religieuse, de négocier sa position dans un contexte global. Cette tension est présente, pour prendre un exemple latino-américain, dans le processus de déterritorialisation des religions afro-brésiliennes, où la reconnaissance de Porto Alegre comme « principale ville de référence » (Oro, 1995 : 72) par les pratiquants du batuque en Argentine et en Uruguay n'empêche pas la prolifération de récits concurrents sur les origines, ni la remise en question de cet ancrage historique et religieux. Stuart Hall (1998 : 32) a justement rejeté la notion de globalisation en tant qu' " espace non contradictoire », montrant qu'il s'agit au contraire d'une notion constamment contestée, où les rapports entre les différents éléments sont sans cesse renégociés.

On comprend la méfiance de certains auteurs à l'égard de l'idée même de déterritorialisation, telle qu'elle est défendue par exemple par Appadurai (1996), puisque, en prônant la disparition de tout ancrage territorial, elle remet en question les fondements mêmes de l'anthropologie. Ainsi, Jackie Assayag écrit à juste titre que, chez Appadurai, l'avènement de l' « écoumène global » obligerait l'anthropologie à penser indépendamment des catégories d'ethnie définie, de territoire délimité, d'identité fixée, mais aussi de culture conçue comme un tout homogène, consistant et autonome, « abandonnant la perspective culturelle selon laquelle normes et valeurs seraient partagées localement par un bloc d'humanité enraciné dans un sol et développant sur cette base une identité primordiale » (1998a : 206). Comment donc continuer à pratiquer l'ethnologie si tous ces objets familiers deviennent évanescents? La réponse d'Assayag est que l'anthropologie d'Appadurai est « sans terrain » (ibid.), cet auteur ayant succombé à la fantaisie d'une économie sans territoire et de populations sans terre : « Ce n'est pas parce que le 'transnational' élude le territoire (à la différence de l'international) qu'il est 'hors lieu'» (ibid. : 213). Cette même critique est portée par Jonathan Friedman à la «vulgate transnationale », qui se caractériserait, selon cet auteur, par l'absence d'ancrages dans la recherche empirique.

Mais peut-on encore de nos jours parler de culture homogène, également distribuée entre les membres d'un même groupe social, ou de catégories ethniques correspondant à des identités figées? Est-ce le résultat d'une nouvelle donne, produit de la « postmodernité », ou quelque chose qui a toujours existé, mais qui rarement a été pris en compte par un observateur - l'anthropologue - souvent en quête d'élégantes systématisations? Dans le cas de mon domaine de recherche, les religions afro-américaines, cela devient évident. Nous savons que le savoir religieux n'est jamais distribué de façon homogène : il est contrôlé et est source de différenciation au sein des groupes de culte. On ne peut donc pas parler de la « culture afro-brésilienne » ou « afro-cubaine » comme d'un ensemble homogène, parce que le savoir - et encore plus le savoir rituel - n'est pas le patrimoine de tout un chacun (cf. Capone, 1999a et Palmié, 2001). C'est la recherche empirique qui nous oblige, au moins dans ce cas, à remettre en question des notions qui sont à la base même de la pratique « traditionnelle » de l'ethnologie. 


\section{DE L'HYBRIDITÉ À LA CRÉOLISATION}

Jonathan Friedman voit dans le travail de Clifford une profonde tension et une ambivalence. D'une part, ce dernier serait fasciné par l'idée d'hybridité, considérée comme une solution à ce qui est perçu comme l'un des grands problèmes du genre humain : l'essentialisme, dans le sens d'une identification collective fondée sur le partage de valeurs et de symboles. De l'autre, ses écrits révéleraient « une conscience, parfois assez aiguë, du pouvoir de l'histoire et des forces qui non seulement dilatent et contractent les empires, mais poussent également tous les gens à l'essentialisme » (2000 : 191). Ainsi, la transnationalisation serait souvent pensée comme un processus nécessairement producteur d' « hybridité ». En termes identitaires, il s'agirait des hyphenated identities, ou « identités à traits d'union » (Assayag et Bénéï, $2000: 18$ ). Or, selon Friedman (1994: 232), " the trans-ethnic is often a weak identity, supported by cultural classifiers, in a more serious context of stronger separate ethnicities in conflict ». Cela relèverait du « discours trans-» qui, selon cet auteur, « consiste le plus souvent en une déconstruction des catégories censées être pures ou homogènes, afin de mettre au jour leur caractère construit. Dans ce type de démarche, il existe une relation logique entre le 'trans-' et l'hybride ou même le créole. Ces deux derniers termes sont utilisés pour décrire des réalités sociales culturellement plurielles ou mixtes, une pluralité qui provient du mouvement de la culture à travers le monde entier » (Friedman, 2000 : 194). Mais, chez les anthropologues, on trouverait aussi un autre modèle, qui affirme que le monde a d'abord été une mosaïque d'unités culturelles distinctes. La mondialisation aurait provoqué l'ouverture de ces unités et la culture circulerait aujourd'hui à travers le monde, créant un processus d'interpénétration culturelle que l'on désigne par les termes d'hybridité ou de créolité, ce que Friedman (1994) appelle « une mosaïque soluble » : « Dans cette dernière approche, les termes 'trans- $\mathrm{X}$ ' + hybridité + mondialisation forment une totalité conceptuelle » (Friedman, $2000: 195)$.

Nous avons vu comment l'idée d'un monde formé de sociétés closes, sans aucun lien entre elles, a été critiquée autant par les tenants du transnationalisme que par ses détracteurs. Mais les critiques adressées à la notion de créolisation se concentrent sur cette supposée ouverture contemporaine d'un monde autrefois compartimenté. Ainsi, Friedman accuse le travail de Hannerz de s'inspirer d'un « essentialisme confus »: "This mingling of cultures, the fusion that leads to supposedly new products, is a metaphor than can only succeed in terms of a previous metaphor, that of culture as matter, in this case, apparently, a fluid. In strictly formal terms this substantialization of culture also leads to an understanding of the latter in terms of products rather than production. Thus, while allusion is made to the 'social organization of meaning', the social organization as such all but disappears in references to flows of meaning, from the center to periphery and back» (Friedman, $1994: 208)^{3}$.

Cette essentialisation de la notion de culture trouverait son expression la plus aboutie dans la notion de créolisation. De nos jours, les remises en question de la pertinence de cette notion se multiplient, notamment chez les linguistes ( $c f$. Harris et

3. Voir aussi Strathern (1992) pour une critique des analyses en termes de créolisation. 
Rampton, 1999). Les mécanismes à l'œuvre dans la production des langues créoles ou du pidgin seraient en réalité communs à l'ensemble des langues dites naturelles. Lorsque l'on passe du domaine de la linguistique à celui de l'anthropologie, cette notion ne peut qu'exprimer, pour Friedman (1994 : 209), l'idée de mélange de deux (ou plus) cultures « pures ». Dans cette approche essentialiste de la culture, un créole serait ainsi le résultat du mélange des cultures « pure noire + pure blanche + pure amérindienne » (ibid.). Cette notion aurait ainsi pour effet d'essentialiser les « cultures ataviques » qui auraient donné naissance aux «cultures créoles ». Cette classification a été produite dans le contexte des plantations, structuré par des rapports de domination. On aimerait ajouter que les choses ne sont pas nécessairement si simples, car les « créoles » étaient aussi les Blancs « purs », nés en terre américaine. En ce sens, la notion de créolité n'impliquait pas nécessairement un mélange « racial », ni culturel.

Dans un texte de 1997, Hannerz reconnaît que le terme « créolisation » est devenu extrêmement ambigu. Trop souvent, dans l'opposition entre les notions de global et de local, le local est associé à la continuité, alors que le global entraînerait le changement. De même, le national serait associé à l'authentique, alors que le transnational donnerait naissance à l'hybride ( $c f$. Clifford, $1997: 10$ ). A partir de mes propres expériences en ce qui concerne la transnationalisation des religions afro-américaines, je pourrais répondre que, au sein du transnational, on rencontre aussi le «pur» et l'« authentique » dans la revendication constante d'une tradition plus proche des origines. Les rapports entre centre et périphérie sont ainsi constamment remis en question et les discours sur la pureté et la fidélité aux traditions réaménagent les rapports entre « terre des origines » et « diaspora ».

\section{LES ÉTUDES AFRO-AMÉRICAINES ET LES THÉORIES SUR LA TRANSNATIONALISATION}

Est-ce un hasard si la plupart des termes utilisés pour parler de transnationalisation sont issus de l'univers afro-américain? Je pense notamment à « créolisation », mais aussi à «hybridité », que Hannerz (1997 : 24) associe au concept d'homme marginal, créé par Robert E. Park (1928), éminent représentant de l'Ecole de sociologie de Chicago. L'Afro-Américain serait l'incarnation de cet homme déchiré entre plusieurs cultures, totalement déraciné, « déterritorialisé », puisqu'il n'a plus d'ancêtres, plus de lieu d'origine défini, si ce n'est dans une Afrique « mythique ». Il serait ainsi une sorte de métaphore de l'homme postmoderne, auquel la religion permet de se « reterritorialiser », en s'inscrivant dans un espace, réel ou mythique, qui dépasse les frontières nationales. La même notion de flux culturels nous rappelle les théories sur l'acculturation, avec ses flux et contre-flux, l'influence du centre sur la périphérie et vice-versa, nouvelle version des mouvements de contre-acculturation. Et si, finalement, on était en train de parler de la même chose? Et si, depuis le début, l'univers afro-américain s'était structuré selon une logique « transnationale » et pas simplement par le déplacement forcé des esclaves africains?

Au moins dans le cas des religions afro-américaines, l'approche transnationale est imposée par les mêmes matériaux ethnographiques. En ce sens, on pourrait dire que l'empirique produit l'idéologique, puisque l'objet est déjà construit de façon « transnationale ». Si l'on peut aujourd'hui discuter la notion d' « écoumène global $»^{4}$, défen- 
due par Hannerz entre autres, on est au contraire obligé de penser les cultures, et surtout les religions afro-américaines, dans un contexte transnational. Et cela, encore une fois, n'est pas quelque chose de nouveau, car l'on peut parler de transnationalisation au sein de ces religions depuis au moins la fin du XIX ${ }^{\mathrm{e}}$ siècle, avec les allers-retours des initiés du candomblé entre le Brésil et l'Afrique. L'étude des religions afro-américaines n'a jamais pu se faire qu'en termes de réseaux, entre l'Afrique et le Brésil par exemple, mais aussi entre les différents centres, détenteurs des traditions africaines sur le sol américain. On passerait ainsi d'une approche comparativiste, qui souligne la continuité culturelle entre l'Afrique et l'Amérique, à une nouvelle approche qui, en prenant en compte l'espace de circulation des acteurs sociaux, des symboles et des savoirs, peut s'avérer particulièrement productive. Cet espace, nous l'avons vu, existe depuis longtemps, mais il n'a jamais eu l'ampleur actuelle. De nos jours, cette nouvelle approche s'impose lorsque l'attention du chercheur se porte sur les efforts de systématisation du savoir religieux par les «élites » des religions afro-américaines : les gardiens de la tradition. Malheureusement, les processus d'incorporation, de réaménagement et d'adéquation aux réalités locales d'un savoir produit dans des forums internationaux - résultat d'un travail d'épuration et de mise en valeur de certains éléments, choisis comme symboles de la «tradition africaine »-n'ont pas encore été analysés avec l'attention qu'ils méritent.

Les recherches sur la transnationalisation des religions afro-américaines peuvent donc apporter, à mon sens, une contribution intéressante au débat sur les théories de la globalisation et de la transnationalisation culturelle. Il faudra montrer comment, dans le domaine afro-américaniste, les théories actuelles sur la globalisation ne font que reprendre ce qui est à la base même de ces études, à savoir la reconfiguration des identités individuelles et collectives au sein d'un monde devenu transnational. Or, la colonisation du Nouveau Monde constitue un des tout premiers cas de globalisation, économique et culturelle. Et force est de constater que la première transnationalisation culturelle, d'une telle envergure, a été entraînée par le déplacement d'esclaves africains ${ }^{5}$.

L'étude des religions afro-américaines offre aussi un exemple de l'enracinement dans le passé des théories qui, aujourd'hui, prônent la « rupture postmoderniste ». Nouvelle preuve que le monde, comme l'affirment les détracteurs des théories sur la globalisation, est «globalisé » depuis fort longtemps. Qu'est-ce qui change aujourd'hui? L'accroissement des liens entre les différents centres, détenteurs des

4. La notion d'écoumène a été développée par Ulf Hannerz (1996 : 7), lequel a repris un terme utilisé par Alfred Kroeber lors de la Huxley Memorial Lecture, qui s'est tenue en 1945 à Londres, au Royal Anthropological Institute. Le terme « écoumène » vient du grec oikoumenê, qui désignait aux yeux des anciens Grecs la totalité du monde connu. Il est utilisé par Hannerz et par d'autres chercheurs ( $c f$. Igor Kopytoff, 1987) pour désigner une « région d'interaction et d'échange culturels ». Sur la notion d'écoumène, voir Kroeber (1945), Hannerz (1996, 1997) et Mintz (1996).

5. Les critiques adressées aux défenseurs des théories sur la globalisation, formulées par Sydney Mintz (1996), ne peuvent pas se limiter, à mon sens, au cas de la Caraïbe, interprétée comme premier « village global » et comme premier « laboratoire de la modernité », mais elles doivent s'élargir à l'ensemble du continent américain. Pour Mintz, ce qui caractérise l'écoumène antillais est le système des plantations, qui a permis l'élaboration de la culture afro-américaine. Le Brésil devrait, tout naturellement, être inclus dans ce même écoumène, ainsi que d'autres anciennes colonies américaines dont l'économie était fondée sur la culture extensive du sucre ou du coton et sur l'exploitation d'une main-d'œuvre esclave. Cet écoumène ne serait alors plus « antillais », mais « américain ». 
traditions africaines, qui ne se situent pas nécessairement en Afrique (comme c'était le cas jusqu'aux années 1970), mais aussi sur le continent américain. Un exemple parmi d'autres est offert par la création d'un « écoumène yoruba », dans un rapport spéculaire entre Afrique et Amérique, mais aussi entre les différents centres producteurs de discours et de pratiques dits traditionnels sur le continent américain (Bahia, La Havane ou Matanzas) ${ }^{6}$. L'anthropologie afro-américaniste n'a jamais été limitée à un territoire et à une idée de culture conçue comme un tout autonome, car il a toujours existé un « ailleurs », réel ou mythique, qu'il fallait prendre en compte. De même, elle n'a jamais pu faire l'économie d'une approche historico-comparative.

$\mathrm{Si}$, pour reprendre ce que dit Jackie Assayag (1998a), «l'idée de globalisation ne [peut] acquérir de finalité cognitive sans une discussion de fond préalable sur ses dimensions historiques ou comparatives, à la fois dans la longue durée et selon une perspective transcontinentale », alors l'apport des études afro-américanistes peut se révéler primordial. On pourra ainsi montrer que, dans le cas des religions afroaméricaines, on a toujours été obligé de penser la multilocalité, par exemple dans la recherche des « africanismes » au sein des cultures américaines, et cela malgré toute dérive culturaliste. Les cultures « afro-américaines » n'ont d'ailleurs jamais été enfermées dans un territoire, elles ont toujours été inscrites dans un « écoumène ». Dans ce domaine, une déterritorialisation est toujours suivie d'une reterritorialisation, réelle ou symbolique, et nous ne sommes pas confrontés à " une anthropologie en termes de non-lieu », comme l'affirme Assayag (1998b) en analysant l'ouvrage de Gupta et Fergurson. De même, le transnational peut être difficilement réduit, comme le suggère Jonathan Friedman, à une idéologie d' " intellectuels en diaspora », dont les recherches n'auraient aucun ancrage dans la recherche empirique et qui ne seraient que la projection de leurs propres expériences d'élites « voyageuses et déracinées ». Dans le cas des religions afro-américaines, ceux qui voyagent - et qui sont symboliquement " déracinés », car les racines sont ailleurs, un ailleurs réel ou mythique - sont les initiés dans ces religions. Le "voyage » est ainsi un élément constitutif de l'objet. En ce sens, roots and routes (les racines et les routes, comme dirait Clifford) ne sont pas en opposition ( $c f$. Friedman, 2000) : à une multiplicité de racines correspond la complexité du déploiement des territoires d'origine et des liens (les allers-retours) entre ceux-ci. Depuis longtemps, dans ce domaine, les racines se sont transformées en rhizomes ${ }^{7}$.

On pourra aussi montrer comment, dans le domaine afro-américaniste, on a toujours été dans le «global», puisque le « local»n'a jamais pu faire l'économie d'un ailleurs (l'Afrique, mais aussi les métropoles coloniales). De plus, l'analyse des religions afro-américaines permet de remettre en question l'opposition entre l'ancrage dans un territoire (le «national») qui permettrait la revendication d'une culture " pure » et « authentique », et la déterritorialisation associée au transnational, qui mettrait en avant une culture « hybride » ou « créolisée ». Dans ce cas, nous l'avons vu, le transnational n'empêche pas la production de discours essentialistes, où

6. Le dernier exemple de cette « globalisation religieuse » a été offert par la réalisation du $8^{\text {ème }}$ Congrès mondial sur la tradition et culture des orisha, du 13 au 17 juillet 2003 à La Havane (Cuba), qui était présenté en anglais comme étant le $8^{\text {th }}$ Global Yoruba Congress.

7. Un excellent exemple est fourni par le passage de la santería à l'orisha-voodoo aux Etats-Unis (Capone, 1999b). 
la culture, en dépit de son évidente transformation et adaptation, est pensée comme « pure » et « traditionnelle ». Ce sont les lieux de référence de cette tradition - de ses racines - qui se multiplient et s'opposent. Ainsi, le transnational n'entraîne pas nécessairement des phénomènes d'hybridation ou de créolisation.

\section{DU GLOBAL AU LOCAL}

L'approche transnationale permet, entre autres, de révéler la nature essentiellement politique de la religion. Le processus de transnationalisation des communautés religieuses se fait, pour la plupart, dans un contexte d'articulation entre pouvoir et prestige qui est souvent laissé de côté dans les analyses ethnographiques. Il conviendrait d'articuler les notions de « global » et de « local », en analysant comment les contenus originellement locaux se transnationalisent pour s'ancrer dans des territoires toujours plus éloignés. Cela montre l'importance du processus d'indigénisation des religions transnationalisées : elles s'adaptent aux différents contextes nationaux, en négociant leur place au sein du champ religieux local et au cœur de la société nationale. La diffusion du batuque et de l'africanismo en Argentine (cf. Frigerio, 1997) ne présente pas les mêmes enjeux que celle de la santería aux Etats-Unis. Les identités religieuses en question, bien qu'elles se réfèrent à une même origine africaine ou " yoruba », doivent être renégociées dans un cadre national avec les autres identités religieuses en présence, tout en se confrontant avec le processus de construction des identités nationales et leurs interactions historiques avec d'autres sociétés nationales. C'est le cas, par exemple, de l'intérêt renouvelé pour la santería cubaine aux EtatsUnis après la révolution castriste. L'indigénisation des religions afro-cubaines entretient ainsi un lien étroit avec l'histoire politique des rapports entre les Etats-Unis et la Cuba post-révolutionnaire ( $c f$. Palmié, 2001 : 4). Il me semble donc indispensable de s'interroger, non seulement sur le processus de transnationalisation des identités religieuses, mais aussi - et surtout - sur leurs processus d'indigénisation. L'analyse des modes d'attribution de nouvelles significations à des éléments issus d'autres contextes, qui doivent s'accorder avec des pratiques et des histoires locales, s'avère alors indispensable.

La complexité croissante des modèles d'interdépendance entre groupes religieux, issus d'un même centre (tel l'EURD pour le pentecôtisme) ou revendiquant une même origine (telles la santería ou la religion des orisha), permet de mettre en évidence le rôle joué par des acteurs transnationaux, sortes de « passeurs » du savoir rituel ou religieux. Cependant, l'analyse des relations transnationales ne fait pas nécessairement l'impasse sur le rôle joué par l'Etat, comme le montre le cas cubain analysé par Kali Argyriadis, où l'Etat manipule des acteurs transnationaux, tel que l'Oni d'Ifé, pour atteindre ses fins.

Les articles réunis dans ce dossier sur la transnationalisation religieuse témoignent de la complexité de ces notions. Pauline Guedj remet en question une théorie très répandue parmi les sociologues des religions, selon laquelle le monde « postmoderne » serait caractérisé par un éclatement des pratiques religieuses et une individualisation des croyances, sans aucun lien avec des institutions religieuses et politiques. Les Etats-Unis sont souvent cités comme l'exemple le plus abouti de cette nouvelle approche religieuse. Pauline Guedj montre comment l'apparition des cultes d'origine africaine aux Etats-Unis, et en particulier la religion akan, est le résultat 
non pas d'un éclatement postmoderne du religieux, mais d'une histoire politique et nationaliste afro-américaine qui a toujours entretenu des rapports étroits avec la religion. La « revitalisation » des religions d'origine africaine aux Etats-Unis doit être ainsi replacée dans le contexte plus large de l'histoire politique afro-américaine et plus particulièrement du nationalisme noir, qui considère que le salut des Noirs réside dans la constitution d'une Nation indépendante, séparée symboliquement de la société blanche environnante. Cette mouvance politique a promu un phénomène de «réafricanisation » au sein de la communauté afro-américaine, avec l'adoption de cultes d'origine africaine, ce qui a entraîné une profonde modification de son discours et de ses fondements idéologiques. Loin d'une quête personnelle du salut ou des origines, ces nouvelles pratiques religieuses, de plus en plus populaires aux Etats-Unis, apparaissent alors comme les pièces maîtresses d'un combat politique contre l'oppression, usant de la formation de réseaux transnationaux entre l'Amérique et l'Afrique comme d'une nouvelle forme de séparatisme. Il est ainsi possible, aujourd'hui, de parler d'un « nationalisme transnational » au sein de la communauté afro-américaine, fondé sur l'idée de l'existence d'ethnies se déployant entre les Etats-Unis et l'Afrique.

Alejandro Frigerio souligne dans son article l'importance de l'origine nationale des pratiquants des religions afro-américaines dans les processus de transnationalisation religieuse. La diffusion de ces religions a entraîné la formation de réseaux de parenté religieuse qui dépassent les frontières nationales pour donner naissance à des communautés transnationales. Initialement constituées par les initiés locaux et leur famille rituelle dans le pays d'origine, tel le batuque introduit en Argentine par des Brésiliens, ces communautés originellement binationales intègrent de nos jours des pratiquants de plusieurs nationalités, souvent issus d'autres variantes nationales des religions afro-américaines. Frigerio analyse le processus de formation de ces communautés, qui passent d'une première phase marquée par des attaches principalement locales, à une deuxième caractérisée par des liens avec une communauté étrangère, pour terminer par leur insertion dans des réseaux transnationaux plus vastes. Frigerio distingue deux types de « diaspora religieuse » : l'une, « primaire », qui fait référence aux communautés de culte les plus anciennes, comme celles du candomblé bahianais ou de la santería cubaine, et l'autre, "secondaire », constituée par les communautés les plus récentes, comme celles du batuque argentin, du candomblé de São Paulo ou de la santería aux Etats-Unis. L'étude des parcours religieux des initiés et des processus de construction de leur carrière religieuse met en évidence des caractéristiques communes à l'ensemble des variantes religieuses de la « diaspora secondaire » : dans la plupart des cas, la reconnaissance sociale conquise dans le pays d'origine doit être regagnée dans le pays d'accueil. Pour cela, certaines modalités de culte, tel l'umbanda en Argentine, semblent exercer un rôle de médiation - véritables « modalités-pont» - vers les variantes plus « africaines », aidant le nouvel adepte à se familiariser avec la vision du monde de ces religions. Dans les pays d'Amérique latine, ces modalités fonctionnent ainsi comme des ponts cognitifs entre le catholicisme populaire et les variantes plus « africaines » des religions afro-américaines.

Nahayeilli Juárez Huet présente les premiers résultats de sa recherche sur la diffusion de la santería dans la ville de Mexico. Les données ethnographiques qu'elle a recueillies montrent comment la santería est aujourd'hui une religion qui dépasse les frontières nationales cubaines, et s'étend à plusieurs pays du continent américain. L'essor de cette religion au Mexique constitue un phénomène récent, bien que les 
premiers pratiquants soient arrivés dans ce pays dans les années cinquante. L'auteur analyse les raisons qui amènent les Mexicains à s'initier dans une religion qui n'a pas de racines dans un pays où l'héritage culturel africain a été complètement oublié. Elle met aussi en évidence l'importance de la nationalité des acteurs sociaux dans la constitution du champ religieux, où Mexicains et Cubains s'opposent, tout en reconnaissant leurs liens de descendance religieuse. On retrouve ainsi dans cet article, le premier publié sur la santería au Mexique, plusieurs questions déjà analysées dans d'autres pays, et notamment aux Etats-Unis : la stigmatisation de la santería en tant que « secte satanique », la condamnation des sacrifices d'animaux, les accusations de mercantilisation du culte, le rôle joué par les médias dans la diffusion et la popularisation de la santería, la remise en question des liens de dépendance entre initiateurs (les Cubains) et initiés (les Mexicains).

Kali Argyriadis et moi-même avons mis en commun nos expériences de terrain respectives à Cuba et aux Etats-Unis, afin de développer une réflexion à deux voix sur la santería cubaine. Nous avons cherché à comprendre les mécanismes d'un phénomène paradoxal, ayant pour dénominateur commun la revendication d'une « tradition yoruba » qui serait le monopole des santeros cubains. Au-delà de la dimension religieuse, c'est la question de la cubanité qui est en jeu, les références à l'Afrique, au castrisme, et plus implicitement au christianisme, servant tour à tour les discours et les accusations des différentes parties. Cette analyse a mis en évidence l'imbrication du politique et du religieux dans deux villes - La Havane et Miami - qui forment un tout difficilement dissociable : les deux « sœurs ennemies » de la cubanía. Les pratiques religieuses de la santería sont le locus où s'articulent des enjeux multiples, en termes de politique interne et de stratégie identitaire nationale. Nous montrons comment l'analyse de la constitution des réseaux transnationaux, qui lient (et opposent) les Cubains de l'île aux Cubains de Miami, nécessite une approche contextuelle fine, qui prenne en compte leur inscription historique et les enjeux économiques, sociaux, identitaires et politiques qui les sous-tendent. Les changements actuels, à Cuba et aux Etats-Unis, sont à comprendre alors en termes de jeu d'échelle : d'une pratique religieuse sans dogmes et sans institutions organisées de façon rigide au niveau local, on passe à une certaine orthodoxisation sous la tutelle de l'Etat, pour parvenir, au niveau régional, à un processus d'institutionnalisation avec la création d'Eglises santeras au sein de la communauté cubaine de Miami. Penser ces changements en termes de réseaux transnationaux peut être particulièrement productif, bien que, dans le cas cubain, l'Etat ne soit pas totalement absent.

Dans son article, Maria Julia Carozzi apporte un contrepoint intéressant aux analyses de la transnationalisation des religions afro-américaines, avec l'étude de l'adaptation locale du mouvement du New Age à Buenos Aires. Ce mouvement se structure autour d'un circuit - un réseau segmenté, polycentrique et intégré -, qui brise les frontières distinguant autrefois de façon claire les religions des thérapies alternatives. Ce mouvement met l'accent sur les rapports avec le cosmos et la nature en vue de procurer bien-être et développement spirituel à chaque individu. En revanche, les relations interpersonnelles doivent être marquées par l'éphémère, afin de désacraliser les liens profonds avec les autres, qui sont la source de déséquilibre émotionnel et énergétique. La circulation entre différents groupes et le contact avec des «maîtres » toujours différents permettent ainsi à l'individu de s'épanouir et de parfaire son existence. L'auteur met en lumière les transformations entraînées par ce mouvement 
dans les classes moyennes urbaines en Argentine, qui substituent aux liens d'amitié et familiaux des relations éphémères, constamment changeantes. Le New Age octroie ainsi un caractère sacré à cette tendance au remplacement continu des interlocuteurs dans les relations sociales, associant cela aux notions de bien-être, de développement spirituel, d'équilibre et de santé.

Ari Pedro Oro ajoute une nouvelle dimension à notre réflexion sur la transnationalisation religieuse, en analysant le processus de diffusion de l'Eglise Universelle du Royaume de Dieu (EURD) dans différents pays. Cette Eglise pentecôtiste brésilienne fondée en 1977 à Rio de Janeiro est aujourd'hui présente dans quatre-vingts pays grâce à sa capacité d'adaptation aux différentes cultures locales. Oro se consacre dans le présent article à l'étude de trois cas emblématiques : l'Argentine, le Portugal et l'Afrique du Sud. Comme pour les religions afro-américaines, la question porte sur la manière dont cette Eglise pentecôtiste se localise et s' " indigénise ». Une nouvelle fois, le rôle joué par la nationalité des acteurs sociaux s'avère fondamental pour la compréhension des enjeux de la transnationalisation. Dans chaque pays, le discours et les pratiques religieuses de l'EURD doivent ainsi s'ajuster aux cultures locales, tout en conservant leur propre ethos.

Pierre-Joseph Laurent, qui a poursuivi sa réflexion sur la transnationalisation $\mathrm{du}$ pentecôtisme en analysant, dans son dernier ouvrage Pentecôtistes du Burkina Faso. Mariage, pouvoir et guérison (2003), l'implantation des Assemblées de Dieu (AD) dans ce pays, s'est prêté au jeu, pas toujours facile, de la critique croisée. Le dossier final est ainsi consacré aux différentes lectures de cet ouvrage par des spécialistes tels que Joël Noret, Sandra Fancello et Frédéric Moens. Joël Noret se propose de prolonger la partie historique de l'ouvrage, intégrant à la discussion d'autres éléments du contexte transnational dans lequel, dès le début, se sont trouvées inscrites les AD du Burkina Faso. La contribution de Sandra Fancello nous aide également à éclairer le processus d'implantation des $\mathrm{AD}$, analysé par Pierre-Joseph Laurent, à travers la comparaison des données sur le Burkina Faso avec les données qu'elle a recueilli au Ghana. Cela nous permet de saisir la portée de ce phénomène de transnationalisation religieuse, au-delà des frontières nationales du Burkina Faso, le Ghana et le Nigeria demeurant les principaux foyers de diffusion des Eglises nouvelles en Afrique. Frédéric Moens apporte le regard d'un sociologue sur ces questions, soulignant l'importance, dans le travail de Laurent, de notions telles que « modernité insécurisée » et « modernité globalisée ». Le dossier sur la transnationalisation du pentecôtisme au Burkina Faso se conclut avec la réponse, en forme d'article, de Pierre-Joseph Laurent, dans laquelle il ajoute des éléments nouveaux qui mettent en lumière la complexité - et les pièges - des analyses des processus de transnationalisation religieuse. Il montre comment la conversion au pentecôtisme et le sentiment d'appartenir à une communauté transnationale qui en découle produisent des ressources inédites pour l'entrée dans ce qu'il définit comme « modernité globalisée ». L'analyse de Pierre-Joseph Laurent met l'accent sur l'importance d'observations de longue durée sur le terrain, seul moyen de saisir les liens très complexes entre le local et le global.

Ce dossier sur la transnationalisation des religions ne fait qu'ouvrir un débat, en l'alimentant en « chair ethnographique ». J'aimerais conclure en affirmant que, s'il est certain que les phénomènes de globalisation ne sont pas nouveaux, la réflexion sur la transnationalisation se justifie, elle, par la constitution même de nos objets ethno- 
graphiques, qui nous confrontent à des terrains éclatés, mais reliés par des réseaux de plus en plus complexes. Aux critiques fustigeant l'absence de travail de terrain dans les ouvrages sur la transnationalisation, on répondra que, si le territoire ne disparaît pas mais se transforme, de même le travail de terrain doit se transformer profondément et se déployer sur plusieurs fronts. Mener des recherches sur la transnationalisation des religions oblige alors à réaliser des enquêtes de terrain « multilocalisées », qui transforment profondément la pratique anthropologique ( $c f$. Marcus, 1995). Voilà, peut-être, un nouveau défi pour l'anthropologie.

\section{RÉFÉRENCES BIBLIOGRAPHIQUES}

Albrow, Martin, 1996, The Global Age. State and Society beyond Modernity, Cambridge : Polity.

Amselle, Jean-Loup

2000, « 'Grand Partage' ou mauvais cadrage? », L'Homme, 156, pp. 207-226.

2001, Branchements. Anthropologie de l'universalité des cultures, Paris : Flammarion.

Appadurai, Arjun, 1996, Modernity at Large. Cultural Dimensions of Globalization, Minneapolis, London : University of Minnesota Press.

AssAYAG, Jackie

1998a, « La culture comme fait social global? Anthropologie et (post)modernité », L'Homme, 148, pp. 201-224.

1998b, Compte-rendu du livre de Gupta et Fergurson, Culture, Power, Place. Explorations in Critical Anthropology, Durham, London : Duke University Press, 1997, L'Homme, 148, pp. 260-261.

AssAyAg, Jackie et BÉNÉï, Véronique, 2000, « A demeure en diaspora. Asie du Sud, Europe, Etats-Unis », L'Homme, 156, pp. 15-28.

Beyer, Peter, 1998, « Global Migrations and the Selective Reimagining of Religions », Horizontes Antropológicos, Porto Alegre, 4(8), pp. 12-33.

CAPONE, Stefania

1999a, La quête de l'Afrique dans le candomblé. Pouvoir et tradition au Brésil, Paris : Karthala. 1999b, «Les dieux sur le Net : l'essor des religions d'origine africaine aux Etats-Unis », L'Homme, 151, pp. 47-74.

2001-2002, «Les pratiques européennes des religions afro-américaines », numéro spécial dirigé par S. Capone, Psychopathologie africaine, vol. XXXI, 1.

Clarke, Kamari Maxine, 2004, Mapping Yorùbá networks. Power and Agency in the Making of Transnational Communities, Durham, N. C. : Duke University Press, sous presse.

Clifford, James, 1997, Routes. Travel and Translation in the Late Twentieth Century, Cambridge, Mass. ; London : Harvard University Press.

Corten, André et Marshall-Fratani, Ruth (éd.), 2001, Between Babel and Pentecost. Transnational Pentecostalism in Africa and Latin America, London : Hurst.

Corten, André et Mary, André (éd.), 2000, Imaginaires politiques et pentecôtistes. Afrique/Amérique latine, Paris : Karthala.

Deleuze, Gilles et Guattari, Félix, 1980, Mille plateaux, Paris : Minuit.

Featherstone, M. (éd.), 1990, Global Culture, Nationalism, Globalism, and Modernity, London : Sage.

FrIEDMAN, Jonathan

1988, « Simplifying the Complexity. Assimilating the Global in a Small Paradise », in Karl F. Olwig et Karin Hastrup (éd.), Sitting Culture. The Shifting Anthropological Object, London, New York : Routledge, pp. 268-291. 
1994, Cultural Identity and Global Process, London : Sage.

2000, « Des racines et (dé)routes. Tropes pour trekkers », L’Homme, 156, pp. 187-206.

Frigerio, Alejandro, 1997, « Estabelecendo pontes : articulação de significados e acomodação social em movimentos religiosos no Cone-Sul », in Ari Pedro Oro et Carlos A. Steil (éd.), Globalização e religião, Petrópolis : Vozes, pp. 153-177.

Glick Schiller, N., Basch, L. et Blanc Szanton, C., 1992, The Transnationalization of Migration. Perspectives on Ethnicity and Race, New York : Gordon \& Breach.

Glick Schiller, N. et Fouron, N., 1990, «'Every-where we go we are in danger': Ti Manno and the emergence of a Haitian transnational identity », American Ethnologist, 17(2), pp. 329-347.

Hall, Stuart, 1998, « The Local and the Global : Globalization and Ethnicity », in Anthony D. King (éd.), Culture, Globalization and the World-System. Contemporary Conditions for the Representation of Identity, Minneapolis : University of Minnesota Press, pp. 19-39.

HANNERZ, Ulf

1992, Cultural Complexity : Studies in the Social Organization of Meaning, New York : Columbia University Press.

1996, Transnational Connections, London, New York : Routledge.

1997, « Fluxos, Fronteiras, Híbridos : palavras-chave da antropologia transnacional », Mana, 3(1), pp. 7-39.

Harris, Roxy et Rampton, Ben, 1999, « Creole Metaphors in Cultural Analysis : The Limits and Possibilities of Sociolinguistic », communication présentée au « Transnational Communities Program Seminar Series 'Considering Creolization' », King's College London.

KEARney, M., 1995, « The Local and the Global. The Anthropology of Globalization and Transnationalism », The Annual Review of Anthropology, 24, pp. 547-565.

KIng, Anthony D. (éd.), 1998, Culture, Globalization and the World-System. Contemporary Conditions for the Representation of Identity, Minneapolis : University of Minnesota Press ( $1^{\text {re }}$ éd. 1997).

Kopytoff, Igor, 1987, « The Internal African Frontier : the Making of African Political Culture », in Igor Kopytoff (éd.), The African Frontier, Bloomington : Indiana University Press.

Kroeber, A. L., 1945, « The Ancient Oikoumenê as an Historic Aggregate », Journal of the Royal Anthropological Institute, 75, pp. 9-20.

Latour, Bruno, 1991, Nous n'avons jamais été modernes. Essai d'anthropologie symétrique, Paris : La Découverte.

Marcus, George, 1995, «Ethnography in/of the World System : the Emergence of Multisited Ethnography », The Annual Review of Anthropology, 24, pp. 95-117.

Mintz, Sidney W., 1996, « Enduring Substances, Trying Theories : the Caribbean Region as Oikoumenê », The Journal of the Royal Anthropological Institute, 2(2), pp. 289-311.

Oro, Ari Pedro, 1995, « A desterritorialização das religiões afro-brasileiras », Horizontes Antropológicos, 3, pp. 69-79.

Oro, Ari Pedro et SteIL, Carlos A. (éd.), 1997, Globalização e religião, Petrópolis : Vozes.

PAlmié, Stephan, 2001, « Of Pharisees and Snark-Hunters : Afro-Cuban Religion as an Object of Knowledge », Culture and Religion, 2(1), pp. 3-19.

PARK, Robert E., 1964 [1928], Race and Culture, New York : Free Press.

Smith, Anthony D., 1998 [1990], « Para uma cultura global?», in Mike Featherstone (éd.), Cultura Global. Nacionalismo, Globalização e Modernidade, Petrópolis : Vozes.

STRATHERn, Marylin, 1992, Reproducing the Future, New York : Routledge.

VEeR, Peter van der, 2001, « Transnational Religion », communication présentée au colloque sur « Transnational Migration : Comparative Perspectives », Princeton University, 30 juin- $1^{\text {er }}$ juillet.

Willaime, J.-P. (éd.), 1999, « Le pentecôtisme : les paradoxes d'une religion transnationale de l'émotion », Archives des sciences sociales des religions, 105. 